Pacific Journal of Mathematics

A MEASURE OF CONVEXITY FOR COMPACT SETS 


\section{A MEASURE OF CONVEXITY FOR COMPACT SETS}

\section{ROLF SCHNEIDER}

For a subset $M$ of $d$-dimensional real vector space $R^{d}$ let

$$
c(M)=\inf \{\lambda \geqq 0 \mid M+\lambda \text { conv } M \text { is convex }),
$$

where conv $M$ is the convex hull of $M$ and denotes vector addition of sets. Among the compact subsets of $R^{d}$, the convex sets are characterized by the equality $c(M)=0$. It is proved that $c(M) \leqq d$ for arbitrary subsets of $R^{d}$, with equality if and only if $M$ consists of $d+1$ affinely independent points. If $M$ is either unbounded or connected, then $c(M) \leqq d-1$; the bound $d-1$ is best possible in either case.

For subsets $M_{1}, M_{2}$ of $d$-dimensional real vector space $R^{d}$, the Minkowski (or vector) sum is defined by

$$
M_{1}+M_{2}=\left\{x_{1}+x_{2} \mid x_{i} \in M_{i}, i=1,2\right\} \text {. }
$$

Minkowski addition plays an essential role in the theory of convex bodies, due to the fact that the sum of convex sets is always convex. On the other hand, the sum $M_{1}+M_{2}$ may be convex without $M_{1}, M_{2}$ being convex; for instance, if $M$ is the boundary of a convex body $K$, then $M+M=K+K$. Moreover, it is easy to see that the sum of an arbitrary subset $M \subset R^{d}$ and a suitable multiple of its convex hull is always convex. This leads us to the definition below. In the following, the abbreviations cl, int, rel int, bd, aff, conv, dim denote, respectively, closure, interior, relative interior, boundary, affine hull, convex hull, dimension.

For a subset $M \subset R^{d}$, define

$$
c(M)=\inf \{\lambda \geqq 0 \mid M+\lambda \operatorname{conv} M \text { is convex }\}
$$

(here $\lambda A=\{\lambda x \mid x \in A\}$ ). The empty set $\varnothing$ is considered as convex, hence $c(\varnothing)=0$. Clearly $M+\lambda \operatorname{conv} M$ is convex for all $\lambda>$ $c(M)$. If we write

$$
M_{\lambda}=(1+\lambda)^{-1}(M+\lambda \operatorname{conv} M),
$$


then $M_{\lambda} \subseteq \operatorname{conv} M \subseteq$ conv $M_{\lambda}$, hence we may also write

$$
c(M)=\inf \left\{\lambda \geqq 0 \mid M_{\lambda}=\operatorname{conv} M\right\} .
$$

Our main object is to prove the following theorem.

THEOREM. For every set $M \subset R^{d}$,

$$
0 \leqq c(M) \leqq d
$$

The equality sign on the left holds if $M$ is convex, and for bounded $M$ it holds only if $\mathrm{cl} M$ is convex. The equality sign on the right holds if and only if $M$ consists of $d+1$ affinely independent points.

Restricted to the family of compact sets, the functional $c$ might, therefore, serve as a "measure of convexity" (or rather "measure of non-convexity", since it is minimal, instead of maximal, for convex sets; but this is immaterial). The term "measure of convexity" is chosen in reminiscence of the "measures of symmetry" for convex bodies, as defined by Grünbaum [2,p.234]. The functional $c$ has certain properties analogous to those which Grünbaum proposes to consider for measures of symmetry, for instance

$$
c(T M)=c(M)
$$

for every $M \subset R^{d}$ and every nonsingular affine transformation $T$ of $R^{d}$. Furthermore, it can be shown that (compare Grünbaum [2,p. 243])

$$
c\left(M_{1}+M_{2}\right) \leqq \max \left\{c\left(M_{1}\right), c\left(M_{2}\right)\right\}
$$

for $M_{1}, M_{2} \subset R^{d}$. However, in contrast to the situation studied by Grünbaum, the following should be pointed out. If \|\| is a Euclidean norm on $R^{d}$, and if the set of nonempty, compact subsets of $R^{d}$ is endowed with the Hausdorff metric defined by

$$
\rho\left(M_{1}, M_{2}\right)=\max \left\{\sup _{x \in M_{1}} \inf _{y \in M_{2}}\|x-y\|, \sup _{x \in M_{2}} \inf _{y \in M_{1}}\|x-y\|\right\}
$$

then $c$ is not continuous, even if restricted to the compact sets with interior points. For instance, take a triangle $T \subset R^{2}$ and replace one of its edges by the two segments which join the endpoints of the edge to an interior point of $T$. The resulting nonconvex quadrangle $Q$, which can be chosen arbitrarily close to $T$ in the Hausdorff metric, has $c(Q)=1$, whereas $c(T)=0$. 
Let us now proceed to the proof of the theorem. We split it into a series of simple propositions, thereby proving some additional results.

First let $M$ consist of $d+1$ affinely independent points; without loss of generality we may assume that $M=\left\{e_{1}, \cdots, e_{d+1}\right\}$, where

$$
\left\langle e_{i}, e_{j}\right\rangle=\left\{\begin{array}{cl}
1, & \text { if } \quad i=j \\
-1 / d, & \text { if } \quad i \neq j
\end{array}\right.
$$

here $\langle$,$\rangle denotes a scalar product on R^{d}$. Writing $T_{i}=$ $\left\{x \in \operatorname{conv} M \mid\left\langle x, e_{i}\right\rangle \geqq 0\right\}$ for $i=1, \cdots, d+1$ we easily see that

$$
e_{i}+d \operatorname{conv} M=(1+d) T_{i}
$$

hence

$$
M+d \operatorname{conv} M=\bigcup_{i=1}^{d+1}(1+d) T_{i}=(1+d) \operatorname{conv} M,
$$

which shows that $c(M) \leqq d$. If, on the other hand, $M+\lambda$ conv $M$ is convex for some $\lambda \geqq 0$, then $0 \in M+\lambda \operatorname{conv} M$, hence $0 \in e_{i}+\lambda \operatorname{conv} M$ for suitable $i \in\{1, \cdots, d+1\}$, from which we deduce that $\lambda \geqq d$. This shows that $c(M)=$ $d$. $d$.

(1) Proposition. $M_{d}=\operatorname{conv} M$ for every set $M \subset R^{d}$, hence $c(M) \leqq$

The following simple proof has been communicated to me by Dr. Wolfgang Weil. It is clear that $M_{d} \subseteq$ conv $M$. Let $x \in(1+d)$ conv $M$. By Carathéodory's theorem, there exists a set $Y \subseteq M$ of affinely independent points such that $x \in(1+d) \operatorname{conv} Y$. Since $c(Y)=\operatorname{dim}$ aff $Y \leqq d$, we have $Y+d$ conv $Y=(1+d)$ conv $Y$, hence

$$
x \in(1+d) \operatorname{conv} Y=Y+d \operatorname{conv} Y \subseteq M+d \operatorname{conv} M
$$

thus conv $M \subseteq M_{d}$, which proves (1).

(2) Proposition. $\quad M_{d-1} \supseteq($ bd conv $M) \cap(\operatorname{conv} M)$ for $M \subset R^{d}$.

Proof. Let $x \in($ bdconv $M) \cap(\operatorname{conv} M)$. The point $x$ lies in a supporting hyperplane $H$ of the convex set conv $M$, hence

$$
x \in H \cap \operatorname{conv} M=\operatorname{conv}(H \cap M)=(H \cap M)_{d-1} \subseteq M_{d-1},
$$

where we have applied (1) to a suitable $R^{d-1}$. 
(3) Proposition. cl $M_{d-1} \supseteq$ bd conv $M$ for $M \subset R^{d}$.

Proof. Let $x \in$ bd conv $M$. Because of (2) we may assume that $x \notin$ conv $M$. Let $U$ be a convex neighborhood of $x$; then $U$ contains a point $y \in$ conv $M$. By Carathéodory's theorem, $y$ is contained in the relative interior of a simplex $S$ whose vertices belong to $M$. If $\operatorname{dim}$ $S<d$, put $H=$ aff $S$ and $z=y$; if $\operatorname{dim} S=d$, let $z \in \operatorname{conv}\{\mathrm{x}, \mathrm{y}\} \cap$ bd $S$ ( $z$ exists because of $x \notin S$ ) and let $H$ be the affine hull of a proper face of $S$ containing $z$. Then $z \in \operatorname{conv}(H \cap M)=(H \cap M)_{d-1} \subseteq M_{d-1}$. Since $z \in U$ and $U$ was arbitrary, we arrive at $x \in \mathrm{cl} M_{d-1}$, which proves (3).

Clearly the index $d-1$ in Proposition (2) or (3) cannot be replaced by a smaller one, as is shown by a set consisting of $d+1$ affinely independent points.

(4) Proposition. If $M \subset R^{d}$ and conv $M \subseteq \mathrm{cl} M_{\lambda}$ for some $\lambda \geqq$ $d-1$, then $c(M) \leqq \lambda$.

Proof. Let $\epsilon>0$. Let $x \in \operatorname{conv} M$. If $x \in$ bd conv $M$, then (2) shows that $x \in M_{d-1} \subseteq M_{\lambda+\epsilon \cdot}$. Suppose, therefore, that $x \in$ int conv $M$. The set $x+\epsilon(1+\lambda)^{-1}(-\operatorname{conv} M+x)$ is a neighborhood of $x$, hence, because of $x \in \operatorname{cl} M_{\lambda}$, it contains a point $y \in M_{\lambda}$. But then we have

$$
\begin{aligned}
x & \in(1+\lambda)(1+\lambda+\epsilon)^{-1} y+\epsilon(1+\lambda+\epsilon)^{-1} \operatorname{conv} M \\
& \subseteq(1+\lambda)(1+\lambda+\epsilon)^{-1}(1+\lambda)^{-1}(M+\lambda \operatorname{conv} M)+\epsilon(1+\lambda+\epsilon)^{-1} \operatorname{conv} M \\
= & M_{\lambda+\epsilon} .
\end{aligned}
$$

We have proved that conv $M \subseteq M_{\lambda+\epsilon}$, hence $c(M) \leqq \lambda+\epsilon$. As $\epsilon>0$ was arbitrary, the assertion (4) follows.

(5) Proposition. $\quad c(M) \leqq d-1$ for every unbounded set $M \subset R^{d}$.

Proof. Let $M \subset R^{d}$ be unbounded. We have to distinguish two cases:

First case. conv $M$ contains a line $L$. Without loss of generality we may assume that $0 \in L$. Let $E$ be a subspace of $R^{d}$ complementary to $L$, and let $\pi$ denote projection on to $E$ in the direction of $L$. Then (e.g., Grünbaum [3,p. 24])

$$
\operatorname{cl} \operatorname{conv} M=(\pi \operatorname{cl} \operatorname{conv} M)+L \text {. }
$$

Let $\lambda>d-1$, and let $x \in \operatorname{conv} M$. Then 


$$
\pi x \in \pi \operatorname{conv} M=\operatorname{conv} \pi M=(\pi M)_{\lambda}
$$

by (1) (applied to $E$ ), hence there exists a point $m \in M$ such that

$$
(1+\lambda) \pi x \in \pi m+\lambda \operatorname{conv} \pi M \text {. }
$$

This yields

$$
\begin{aligned}
(1+\lambda) x & \in(1+\lambda)(\pi x+L) \subseteq \pi m+\lambda \operatorname{conv} \pi M+L \\
& =m+\lambda \operatorname{conv} \pi M+L \quad(\text { since } \pi m+L=m+L) \\
& \subseteq \mathrm{cl} M+\lambda(\pi \operatorname{cl} \operatorname{conv} M+L) \\
& =\operatorname{cl} M+\lambda \operatorname{cl} \operatorname{conv} M \subseteq(1+\lambda) \operatorname{cl} M_{\lambda} .
\end{aligned}
$$

We have proved that conv $M \subseteq \operatorname{cl} M_{\lambda}$, hence (4) implies $c(M) \leqq$ $\lambda$. As $\lambda>d-1$ was arbitrary, we deduce $c(M) \leqq d-1$.

Second case. conv $M$ does not contain a line. Let $\lambda>d-1$, and let $x \in \operatorname{conv} M$. If $x \in$ bd conv $M$, then $x \in \operatorname{cl} M_{d-1}$ by (3). Suppose, therefore, that $x \in$ int conv $M$. Since conv $M$ is unbounded, there exists a direction of infinity, that is, a vector $u \neq 0$ such that $z+\alpha u \in \mathrm{cl}$ conv $M$ for all $z \in \mathrm{cl}$ conv $M$ and all $\alpha \geqq 0$ (e.g., Grünbaum [3, p. 23]). Since conv $M$ does not contain a line, the halfline $\{x-\alpha u \mid \alpha \geqq 0\}$ contains a (unique) point $y \in$ bd conv $M$. By (3) we have $y \in \mathrm{cl} M_{d-1}$, hence any given neighborhood $U$ of $y$ contains a point $z \in M_{d-1}$. The halfline $L_{z}=\{z+\alpha u \mid \alpha \geqq 0\}$ is contained in cl conv $M$, hence

$$
\begin{aligned}
& L_{z} \subset z+(\lambda-d+1)(1+\lambda)^{-1}(\operatorname{cl} \operatorname{conv} M-z) \\
& \quad \subseteq \operatorname{cl}\left[d(1+\lambda)^{-1} M_{d-1}+(\lambda-d+1)(1+\lambda)^{-1} \operatorname{conv} M\right] \\
& \quad=\operatorname{cl} M_{\lambda} .
\end{aligned}
$$

Since the neighborhood $U$ may be chosen arbitrarily small, we see that $x \in \operatorname{cl} M_{\lambda}$. We have proved that $\operatorname{conv} M \subseteq \operatorname{cl} M_{\lambda}$, hence (4) yields $c(M) \leqq \lambda$. As $\lambda>d-1$ was arbitrary, Proposition (5) is proved.

Clearly, the bound $d-1$ in (5) cannot be replaced by a smaller number: If $M$ consists of $d$ parallel lines, or halflines, and aff $M=R^{d}$, then $c(M)=d-1$.

(6) Proposition. For bounded $M \subset R^{d}, c(\mathrm{cl} M) \leqq c(M)$.

Proof. It is well known that $\operatorname{cl} A+\operatorname{cl} B \subseteq \operatorname{cl}(A+B)$ and conv cl $A \subseteq$ cl conv $A$ for arbitrary $A, B \subset R^{d}$, and that these relations 
hold with the equality sign if $A$ is bounded. We deduce that (cl $M)_{\lambda} \subseteq$ $\operatorname{cl} M_{\lambda}$ in general, and that $(\operatorname{cl} M)_{\lambda}=\operatorname{cl} M_{\lambda}$ for bounded $M$. Now if $\lambda>c(M)$, then $M_{\lambda}=\operatorname{conv} M$ and hence $(\operatorname{cl} M)_{\lambda}=\operatorname{cl} M_{\lambda}=\operatorname{cl} \operatorname{conv} M=$ conv cl $M$, so that $c(\mathrm{cl} M) \leqq \lambda$. The assertion follows.

The example of a triangle from which the relative interior of an edge has been omitted, shows that $c(\mathrm{cl} M)<c(M)$ is possible. However, the following holds true.

(7) Proposition. If $c(M)>d-1$, then $c(\mathrm{cl} M)=c(M)$.

Proof. Let $M \subset R^{d}$ be a set with $c(M)>d-1$. If $c(\mathrm{cl} M)<$ $c(M)$, we may choose $\lambda>d-1$ with $c($ cl $M)<\lambda<c(M)$. Then we have

$$
\operatorname{conv} M \subseteq \operatorname{conv} \operatorname{cl} M=(\operatorname{cl} M)_{\lambda} \subseteq \operatorname{cl} M_{\lambda}
$$

and (4) gives $c(M) \leqq \lambda$, a contradiction.

(8) Proposition. If $M \subset R^{d}$ is bounded and $c(M)=0$, then $\mathrm{cl} M$ is convex.

Proof. Let $c(M)=0$. First suppose that $M$ is compact. Let $\lambda>0$, and let $x \in$ conv $M$. Then $x \in M_{\lambda}$, hence $x=(1+\lambda)^{-1}\left(m_{\lambda}+\lambda y\right)$ with suitable $m_{\lambda} \in M$ and $y \in$ conv $M$, which implies

$$
m_{\lambda} \in x+\lambda(-\operatorname{conv} M+x) .
$$

Since $\lambda>0$ may be chosen arbitrarily small and since conv $M$ is bounded, we see that $x \in \mathrm{cl} M$; hence $M=\operatorname{conv} M$. If $M$ is bounded, but not necessarily closed, we have $c(\operatorname{cl} M) \leqq c(M)=0$, hence $\operatorname{cl} M$ is convex.

It is now clear that in order to complete the proof of the theorem, it only remains to prove the following.

(9) PROPOSITION. If $M \subset R^{d}$ is compact and $c(M)=d$, then $M$ consists of $d+1$ affinely independent points.

Proof. Let $M$ be compact and such that $c(M)=d$. By (1) (applied to $R^{d-1}$ ) $M$ cannot be contained in a hyperplane, hence conv $M$ has interior points. Write

$$
R_{\lambda}=\operatorname{conv} M \backslash M_{\lambda} .
$$

We assert that 


$$
\operatorname{cl} R_{\lambda} \subset \text { int conv } M \quad \text { for } \lambda>d-1
$$

For the proof let $x \in$ bd conv $M$. By (2) we have $x \in M_{d-1}$. If $\alpha=(\lambda-d+1)(1+\lambda)^{-1}$, then

$$
x+\alpha(\operatorname{conv} M-x) \subseteq(1-\alpha) M_{d-1}+\alpha \operatorname{conv} M=M_{\lambda},
$$

hence

$$
R_{\lambda} \subseteq \operatorname{conv} M \backslash \bigcup_{x \in \operatorname{bdconv} M}(x+\alpha(\operatorname{conv} M-x)]
$$

which proves (10) because of $\alpha>0$.

If $\mu>\lambda$, then

$$
\begin{aligned}
M_{\mu} & =(1+\mu)^{-1} M+(\mu-\lambda)(1+\mu)^{-1}(1+\lambda)^{-1} \operatorname{conv} M+\lambda(1+\lambda)^{-1} \operatorname{conv} M \\
& \supseteq(+\mu)^{-1} M+(\mu-\lambda)(1+\mu)^{-1}(1+\lambda)^{-1} M+\lambda(1+\lambda)^{-1} \operatorname{conv} M \\
& =M_{\lambda}
\end{aligned}
$$

hence $\lambda<\mu$ implies $R_{\lambda} \supseteq R_{\mu}$. Since the sets cl $R_{\lambda}$ are compact and nonempty for $\lambda<d$, there exists a point

$$
z \in \bigcap_{0<\lambda<d} \operatorname{cl} R_{\lambda}
$$

By (10), $z \in$ int conv $M$; but

$$
z \notin \operatorname{int}(1+d)^{-1}(m+d \operatorname{conv} M) \quad \text { for } m \in M,
$$

since otherwise for sufficiently large $\lambda<d$,

$$
z \in \operatorname{int}(1+\lambda)^{-1}(m+\lambda \operatorname{conv} M) \subset \operatorname{int} M_{\lambda}
$$

which implies $z \notin \mathrm{cl} \boldsymbol{R}_{\lambda}$, a contradiction.

By Carathéodory's theorem there exists an affinely independent set $Y \subseteq M$ such that $z \in$ conv $Y$, and some subset $Y^{\prime} \subseteq Y$ satisfies $z \in$ rel int conv $Y^{\prime}$. If $\operatorname{dim}$ aff $Y^{\prime}<d$, then every point of rel int conv $Y^{\prime}$ is contained in rel int $(1+d)^{-1}\left(y+d\right.$ conv $\left.Y^{\prime}\right)$ for suitable $y \in Y^{\prime}$. Since $z \in$ int conv $M$, we must have rel int conv $Y$, $\subset$ int conv $M$, from which we deduce that $z \in$ int $(1+d)^{-1}(y+d \operatorname{conv} M)$, which contradicts (11). Hence $Y$ is the set of vertices of a $d$-simplex $S$. 
The only interior point of $S$ which is not contained in

$$
\bigcup_{y \in Y} \operatorname{int}(1+d)^{-1}(y+d S)
$$

is the centroid of $S$. Hence, according to (11), $z$ is the centroid of the simplex $S$. If $M$ contains a point $m \notin Y$, we can replace an appropriate point of $Y$ by $m$ to obtain an affinely independent set $\bar{Y} \subseteq M$ which also satisfies $z \in \operatorname{conv} \bar{Y}$. By the argument above it follows that $\bar{Y}$ is the set of vertices of a $d$-simplex, of which $z$ is the centroid. Since $Y$ and $\bar{Y}$ differ in precisely one point, this is impossible. Hence $M=Y$, which completes the proof.

REMARK. There are many closed unbounded sets $M \subset R^{d}$ which satisfy $c(M)=0$, but are not convex; for instance, in $R^{1}$ the set of all integer points, or in the plane a parabola. Hence it is only for compact sets $M$ that $c(M)$ measures, in some sense, the nonconvexity of $M$.

Remark. Since Proposition (1) is an immediate consequence of Carathéodory's theorem, it is clear that an improvement of this theorem for special sets may yield a corresponding improvement of (l). In particular, the following holds true (for the required variant of Carathéodory's theorem see Danzer, Grünbaum and Klee [1, p. 117] and the references given there).

(12) Proposition. Suppose the set $M \subset R^{d}$ is the union of at most $d$ connected sets or is compact and the union of at most $d$ convexly connected sets; then $c(M) \leqq d-1$.

The bound $d-1$ cannot be replaced by a smaller number, even if one assumes that $M$ is compact and connected: The union of all those edges of a $d$-simplex which contain a specified vertex of the simplex provides a counterexample.

Remark. For convex bodies $K \subset R^{d}$ we could also study the derived functional $b$ defined by $b(K)=c($ bd $K)$. A moment's reflection shows that this is a well-known functional, namely the so-called Minkowski measure of symmetry (Grünbaum [2, p. 246]). Hence we have $d^{-1} \leqq c($ bd $K) \leqq 1$, with equality on the left if and only if $K$ is a simplex, and equality on the right if and only if $K$ has a centre of symmetry. 


\section{REFERENCES}

1. L. Danzer, B. Grünbaum, and V. Klee, Helly's theorem and its relatives, Proc. Symposia Pure Math., vol. VII, Amer. Math. Soc., Providence, (1963), 101-180.

2. B. Grünbaum, Measures of symmetry for convex sets, Proc. Symposia Pure Math., vol. VII, Amer. Math. Soc., Providence, (1963), 233-270.

3. B. Grünbaum, Convex Polytopes, J. Wiley and Sons, 1967.

Received June 25, 1974.

MATHEMATISCHES INSTITUT DER UNIVERSITÄT

D-78 Freiburg i. Br., Germany 



\section{PACIFIC JOURNAL OF MATHEMATICS}

\section{EDITORS}

RICHARD ARENS (Managing Editor)

University of California

Los Angeles, California 90024

\section{J. DugundJI}

Department of Mathematics University of Southern California Los Angeles, California 90007

D. Gilbarg and J. Milgram

Stanford University

Stanford, California 94305

\section{ASSOCIATE EDITORS}
E. F. BECKENBACH
B. H. NeumanN
F. WoLF
K. YoshiDA

\section{SUPPORTING INSTITUTIONS}

\author{
UNIVERSITY OF BRITISH COLUMBIA \\ CALIFORNIA INSTITUTE OF TECHNOLOGY \\ UNIVERSITY OF CALIFORNIA \\ MONTANA STATE UNIVERSITY \\ UNIVERSITY OF NEVADA \\ NEW MEXICO STATE UNIVERSITY \\ OREGON STATE UNIVERSITY \\ UNIVERSITY OF OREGON \\ OSAKA UNIVERSITY
}

\author{
UNIVERSITY OF SOUTHERN CALIFORNIA \\ STANFORD UNIVERSITY \\ UNIVERSITY OF TOKYO \\ UNIVERSITY OF UTAH \\ WASHINGTON STATE UNIVERSITY \\ UNIVERSITY OF WASHINGTON \\ AMERICAN MATHEMATICAL SOCIETY
}

The Supporting Institutions listed above contribute to the cost of publication of this Journal, but they are not owners or publishers and have no responsibility for its contents or policies.

Mathematical papers intended for publication in the Pacific Journal of Mathematics should be in typed form or offset-reproduced (not dittoed), double spaced with large margins. Underline Greek letters in red, German in green, and script in blue. The first paragraph or two must be capable of being used separately as a synopsis of the entire paper. Items of the bibliography should not be cited there unless absolutely necessary, in which case they must be identified by author and Journal, rather than by item number. Manuscripts, in duplicate, may be sent to any one of the four editors. Please classify according to the scheme of Math. Reviews, Index to Vol. 39. All other communications should be addressed to the managing editor, or Elaine Barth, University of California, Los Angeles, California, 90024.

100 reprints are provided free for each article, only if page charges have been substantially paid. Additional copies may be obtained at cost in multiples of 50 .

The Pacific Journal of Mathematics is issued monthly as of January 1966. Regular subscription rate: $\$ 72.00$ a year (6 Vols., 12 issues). Special rate: $\$ 36.00$ a year to individual members of supporting institutions.

Subscriptions, orders for back numbers, and changes of address should be sent to Pacific Journal of Mathematics, 103 Highland Boulevard, Berkeley, California, 94708.

PUBLISHED BY PACIFIC JOURNAL OF MATHEMATICS, A NON-PROFIT CORPORATION Printed at Jerusalem Academic Press, POB 2390, Jerusalem, Israel.

$$
\begin{gathered}
\text { Copyright (C) } 1975 \text { Pacific Journal of Mathematics } \\
\text { All Rights Reserved }
\end{gathered}
$$




\section{Pacific Journal of Mathematics}

\section{Vol. 58, No. $2 \quad$ April, 1975}

Zvi Artstein and John Allen Burns, Integration of compact set-valued functions . . . . . . . . . 297

Mark Benard, Characters and Schur indices of the unitary reflection group $[321]^{3} \ldots \ldots \ldots . .309$

Simeon M. Berman, A new characterization of characteristic functions of absolutely continuous

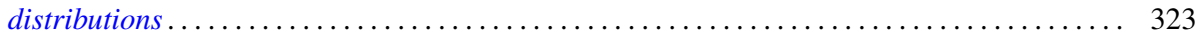

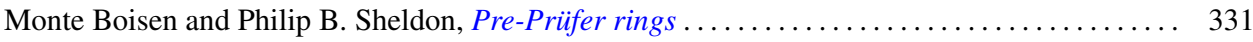

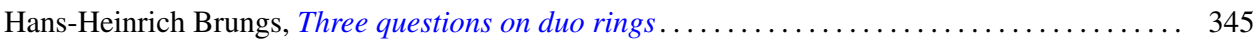

Iracema M. Bund, Birnbaum-Orlicz spaces of functions on groups................. 351

John D. Elwin and Donald R. Short, Branched immersions between 2-manifolds of higher

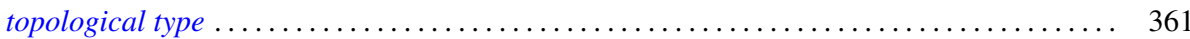

Eric Friedlander, Extension functions for rank 2, torsion free abelian groups . .......... 371

Jon Froemke and Robert Willis Quackenbush, The spectrum of an equational class of

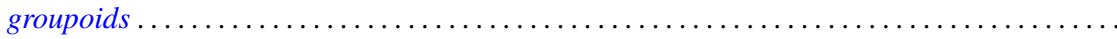

Barry J. Gardner, Radicals of supplementary semilattice sums of associative rings ...........

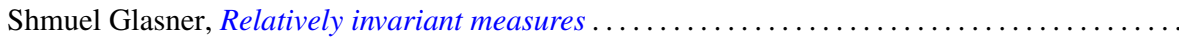

George Rudolph Gordh, Jr. and Sibe Mardesic, Characterizing local connectedness in inverse

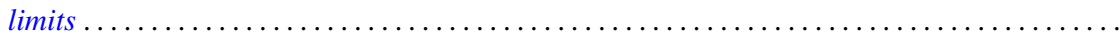

Siegfried Graf, On the existence of strong liftings in second countable topological spaces......

Stanley P. Gudder and D. Strawther, Orthogonally additive and orthogonally increasing

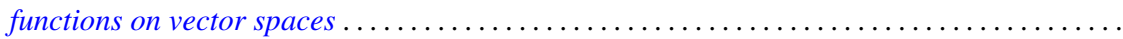

Darald Joe Hartfiel and Carlton James Maxson, A characterization of the maximal monoids and

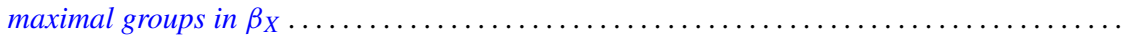

Robert E. Hartwig and S. Brent Morris, The universal flip matrix and the generalized faro-shuffle. .

William Emery Haver, Mappings between ANRs that are fine homotopy equivalences. .

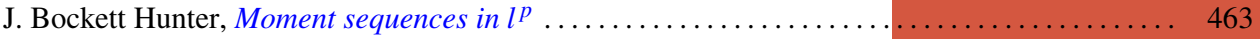

Barbara Jeffcott and William Thomas Spears, Semimodularity in the completion of a poset.... 467

Jerry Alan Johnson, A note on Banach spaces of Lipschitz functions . . . . . . . . . . . . 475

David W. Jonah and Bertram Manuel Schreiber, Transitive affine transformations on

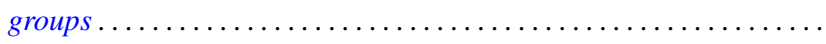

Karsten Juul, Some three-point subset properties connected with Menger's characterization of

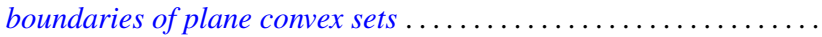

Ronald Brian Kirk, The Haar integral via non-standard analysis . . . . . . . . . . . . . 517

Justin Thomas Lloyd and William Smiley, On the group of permutations with countable

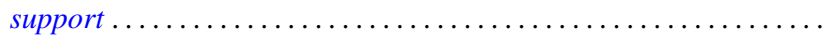

Erwin Lutwak, Dual mixed volumes .................................. 531

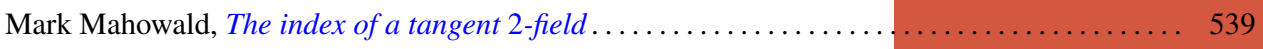

Keith Miller, Logarithmic convexity results for holomorphic semigroups . . . . . . . . . . . . 549

Paul Milnes, Extension of continuous functions on topological semigroups . . . . . . . . . . 553

Kenneth Clayton Pietz, Cauchy transforms and characteristic functions ................ 563

James Ted Rogers Jr., Whitney continua in the hyperspace $C(X) \ldots \ldots \ldots \ldots \ldots \ldots \ldots \ldots .569$

Jean-Marie G. Rolin, The inverse of a continuous additive functional . . . . . . . . . . . . 585

William Henry Ruckle, Absolutely divergent series and isomorphism of subspaces . ........ 605

Rolf Schneider, A measure of convexity for compact sets . ..................... 617

Alan Henry Schoenfeld, Continous measure-preserving maps onto Peano spaces .......... 627

V. Merriline Smith, Strongly superficial elements .......................... 643

Roger P. Ware, A note on quadratic forms over Pythagorean fields . . . . . . . . . . . . . . 651

Roger Allen Wiegand and Sylvia Wiegand, Finitely generated modules over Bezout rings . . . . 655

Martin Ziegler, A counterexample in the theory of definable automorphisms . . . . . . . . . 665 